\title{
Falem bem ou falem mal, mas falem de mim: relação entre trabalho e qualidade de vida do Agente Comunitário de Saúde
}

Any publicity is good publicity: relationship between work and quality of life of the Community Health Agent

Hablar bien o hablar malo, pero hablarme: relación entre el trabajo y la calidad de vida del Agente de Salud Comunitario

Tiago Pereira de Souza ${ }^{1}$, Paulo Antonio Barros Oliveira ${ }^{2}$

${ }^{1}$ Programa de Pós-Graduação em Educação em Ciências: Química da Vida e Saúde da Universidade Federal do Rio Grande do Sul . Porto Alegre-Rio Grande do Sul

${ }^{2}$ Departamento de Medicina Social da Universidade Federal do Rio Grande do Sul Porto Alegre-Rio Grande do Sul

\section{RESUMO}

Este estudo objetivou estabelecer a relação entre o processo de trabalho e a qualidade de vida do grupo de agentes comunitários de saúde do município de Campo Bom, no Vale dos Sinos na região metropolitana de Porto Alegre. Se configurou do tipo descritivo exploratório, transversal, de abordagem quantitativa. Participaram desta pesquisa 62 Agentes Comunitários de Saúde, com faixa etária entre 23 e 61 anos, sendo 58 (93,5\%) mulheres e 4 (6,5\%) de homens. Os participantes responderam um questionário sobre dados sociodemográficos, socioeconômicos e de trabalho, e ao questionário WHOQOL-BREF. A execução desta pesquisa pôde confirmar

Autor de Correspondência:

*Tiago Pereira de Souza. E-mail: tiago.ps.fono@gmail.com 
estatisticamente o impacto do processo de trabalho nos diferentes domínios da qualidade de vida.

Palavras-chave: Agentes Comunitários de Saúde. Saúde do Trabalhador. Qualidade de Vida.

\begin{abstract}
This study aimed to establish the relationship between the work process and the quality of life of the group of community health agents in the city of Campo Bom, in Vale dos Sinos in the metropolitan region of Porto Alegre. This is an exploratory, descriptive, cross-sectional study, with quantitative approach. Sixty-two Community Health Agents, aged 23 to 61 years, participated in this research, with 58 (93.5\%) women and 4 (6.5\%) men. Participants answered a questionnaire on sociodemographic, socioeconomic and work data and the WHOQOL-BREF questionnaire. This research could statistically confirm the impact of the work process in the different domains of quality of life.
\end{abstract}

Keywords: Community Health Workers. Occupational Health. Quality of Life.

\title{
RESUMEN
}

Este estudio tuvo como objetivo establecer la relación entre el proceso de trabajo y la calidad de vida del grupo de agentes comunitarios de salud en la ciudad de Campo Bom, en Vale dos Sinos, en la región metropolitana de Porto Alegre. Se configuró como descriptivo exploratorio, transversal, con enfoque cuantitativo. Sesenta y dos agentes comunitarios de salud, de 23 hasta 61 años, participaron en esta investigación, 58 (93.5\%) mujeres y 4 (6.5\%) hombres. Los participantes respondieron un cuestionario sobre datos sociodemográficos, socioeconómicos y laborales y el cuestionario WHOQOL-BREF. La ejecución de esta investigación podría confirmar estadísticamente el impacto del proceso de trabajo en los diferentes dominios de calidad de vida.

Palabras clave: Agentes Comunitarios de Salud. Salud Laboral. Calidad de Vida.

\section{INTRODUÇÃO}

A cooperação no campo da saúde coletiva dos conhecimentos referentes à questão do trabalho na atenção primária, mais especialmente na função do Agente Comunitário de Saúde (ACS), corrobora com a ampliação da compreensão das relações entre o trabalho e o processo saúde-doença de diferentes cenários de pesquisa, contribuindo de forma significativa, com o entendimento das possíveis 
mudanças na vida dos indivíduos trabalhadores ${ }^{1}$.

Consolidada pela Lei $\mathrm{n}^{0}$ 11.350, de 5 de outubro de 2006, a função dos indivíduos neste momento analisados indica uma transformação na maneira de cuidado constituído na atenção primária, produzindo interdependência entre usuários e a comunidade adstrita $^{2}$. Operam influência recíproca na qualidade de equipe e população das áreas de abrangência da Estratégia de Saúde da Família (ESF), determinando uma política de independência e interrupção do modelo biomédico centralizado na enfermidade, uma vez que apresentam um processo de cuidado com finalidades determinadas, apontadas à promoção da saúde e à prevenção de doenças ${ }^{3}$.

O papel de mediador consolida a principal característica dos ACSs, visto que são trabalhadores com experiências cotidianas capazes de oferecer contrapontos importantes no cuidado dos usuários. São articuladores em potencial das ações em saúde e oferecem para as equipes um "olhar" diferenciado da lógica cartesiana e mecanicista tradicionalmente atribuída à atual configuração da formação em saúde, pois, por estarem inseridos e vivenciarem dia após dia as movimentações comunitárias, possuem um entendimento ampliado da forma como se organizam as relações sociais, culturais, econômicas e ambientais do campo de trabalho da atenção primária ${ }^{3}$.

As diretrizes ministeriais que orientam o trabalho do ACS atentam para a execução supervisionada de atividades de prevenção de doenças e promoção da saúde, por meio de ações domiciliares ou comunitárias, individuais ou coletivas, desenvolvidas de acordo com as diretrizes do Sistema Único de Saúde (SUS). Tais ações configuram-se por meio de instrumentos para diagnóstico demográfico e sociocultural da comunidade, da promoção de ações de educação para a saúde individual e coletiva, do registro (para fins exclusivos de controle e planejamento) das ações de saúde, nascimentos, óbitos, doenças e outros agravos à saúde, do estímulo à participação da comunidade nas políticas públicas voltadas para a área da saúde, da realização de visitas domiciliares periódicas para monitoramento de situações de risco à família e da participação em ações que fortaleçam os elos entre o setor saúde e outras políticas que promovam a qualidade de vida ${ }^{2}$.

Ao falar de subjetividade, faz-se necessária a reflexão sobre "processo de trabalho", de forma a validar a concepção de que trabalhar não se constitui apenas na execução de tarefas e representações práticas da efetivação de ordens e normas, mas em uma sequência de atos e reflexões intelectuais capazes de transformar a natureza e, paralelamente, o próprio trabalhador. Tal aspecto é o que diferencia o trabalho humano do dia a dia da capacidade primitiva dos animais de se adaptar a diferentes situações (por meio de seu instinto de sobrevivência), ou seja, se o trabalho for pensado enquanto atividade cognitiva, pode-se entender o resultado deste como o produto de um planejamento, previamente idealizado pelo trabalhador ${ }^{4}$.

A constituição de cada sujeito trabalhador, composta por perspectivas e idealizações, ao se chocar com um processo de trabalho que ignora tal subjetividade exerce uma ação específica capaz de produzir sofrimento, fazendo com que os coletivos criem estratégias conjuntas para superar esse sofrimento correlato à realização do trabalho ${ }^{1,5}$.

Para o presente projeto é significativa a compreensão de que os ACSs são trabalhadores da saúde, com diretrizes de trabalho preestabelecidas legalmente e suscetíveis ao processo saúde-doença oriundo do trabalho. Dessa forma, a psicodinâmica do trabalho busca o entendimento desse processo, bem como alternativas de intervenção. Trata-se de ações transformadoras e que visam a apropriação da dimensão humana do trabalho por meio de uma perspectiva diferenciada, na qual podemos entender o desfecho das trajetórias de cada indivíduo trabalhador ${ }^{6}$.

Essa filosofia humanística e interdisciplinar carrega 
consigo a aplicação concreta de objetivos claros no que se refere à proposição de mudanças no trabalho, a fim de produzir ambientes favoráveis à satisfação dos trabalhadores, colocando o homem no centro de suas preocupações, buscando o aumento da produtividade organizacional, e caracterizando um dos conceitos de qualidade de vida no trabalho $(\mathrm{QVT})^{7,8,9}$.

A ideia de que a satisfação no trabalho compõe grande parcela da satisfação global, ou seja, na vida do trabalhador ${ }^{10}$, justifica a crescente aplicação de ações voltadas à QVT, visando o resgate de certos valores ambientais e humanos negligenciados pelas sociedades industriais em favor do avanço tecnológico, da produtividade e do crescimento econômico. O sofrimento por conta do trabalho emerge no momento em que os trabalhadores se deparam com configurações fragmentadas, desconexas, burocráticas, normatizadas, rotineiras, carregadas de exigências, que vão de encontro com a sua realidade extralaboral ${ }^{11}$.

Os sujeitos são singulares e apresentam demandas subjetivas antes mesmo de entrarem no mundo do trabalho, não as abandonando no momento de seu ingresso ${ }^{12}$. Pode-se dizer, portanto, que a personalidade dos indivíduos não pode ser totalmente integrada ao trabalho, pois está interligada simultaneamente a diferentes grupos (família, religião, entidades de classe etc.). Tais grupos certamente se constituem em fontes de subjetividade em detrimento do trabalho, sendo que este possui caráter mecanicista e econômico, dificultando ao homem externar sua totalidade como pessoa ${ }^{13}$.

Esta pesquisa surge a partir de uma demanda inicialmente oriunda das práticas no SUS, em decorrência da experiência do pesquisador em processo de formação (residência multiprofissional) e em atuação profissional (educação permanente em saúde), que durou aproximadamente cinco anos. Durante esse período, houve a possibilidade de convívio diretamente com os trabalhadores da atenção básica, sobretudo com ACSs, o que levou o pesquisador a observar de forma empírica a grande quantidade de trabalhadores em sofrimento físico e psíquico. Dessa forma, a pesquisa objetivou estabelecer a relação entre o processo de trabalho e a qualidade de vida do grupo de agentes comunitários de saúde do município de Campo Bom, no Vale dos Sinos, região metropolitana de Porto Alegre.

\section{MÉTODO}

Este estudo foi desenvolvido na cidade de Campo Bom, município brasileiro no estado do Rio Grande do Sul que pertence à Região Metropolitana de Porto Alegre e ao chamado Vale do Rio dos Sinos. A cidade possui $60 \mathrm{~km}^{2}$ de área, 63.767 habitantes e 98,13\% de cobertura da ESF. As unidades de ESF são: Aurora, 25 de Julho, Imigrante, Operária, Quatro Colônias, Porto Blos, Rio Branco e Santa Lúcia.

Trata-se de um estudo do tipo descritivo exploratório, transversal, de abordagem quantitativa. Os participantes foram os trabalhadores Agentes Comunitários de Saúde que exerciam suas atividades nas Unidades de Estratégia Saúde da Família, com exceção dos trabalhadores em férias ou afastados do trabalho no período da coleta dos dados.

O estudo foi organizado dentro do programa de educação permanente (PEP) e faz parte de um projeto "maior" que engloba análises quali-quantitativas, oriundo de uma parceria entre a Prefeitura e o Programa de Pós-Graduação em Saúde Coletiva da Universidade Federal do Rio Grande do Sul (PPGCOLUFRGS), com aprovação sob o número 1.541 .369 no Comitê de Ética em Pesquisa (CEP) da instituição. Os trabalhadores receberam esclarecimentos quanto aos objetivos do estudo e sua condução e, nessa ocasião, todos se dispuseram a assinar o Termo de Consentimento Livre e Esclarecido, ou seja, não houve sujeitos que se negaram a participar.

Inicialmente os participantes responderam a um 
questionário sobre dados sociodemográficos, socioeconômicos e referentes ao trabalho. Posteriormente responderam ao questionário WHOQOL-BREF, desenvolvido pelo Grupo de Qualidade de Vida da Organização Mundial de Saúde (versão abreviada do WHOQOL-100). Nesse instrumento há 26 questões divididas em quatro domínios: físico, psicológico, relações sociais e meio ambiente.

As variáveis quantitativas foram descritas por média e desvio padrão ou mediana e amplitude interquartílica. As variáveis categóricas foram descritas por frequências absolutas e relativas.

Para comparar médias, foram aplicados os testes t-Student ou a Análise de Variância (ANOVA) oneway, complementada por Tukey.

A associação entre as variáveis contínuas e ordinais foi avaliada pelos coeficientes de correlação de Pearson (distribuição simétrica) ou de Spearman (distribuição assimétrica).

A comparação entre os escores médios dos quatro domínios do WHOQOL-BREF foi realizada pela Análise de Variância (ANOVA) para medidas repetidas, complementada pelo teste de Bonferroni.

Para controle de fatores confundidores, a análise multivariada de Regressão Linear foi utilizada. As variáveis que apresentaram uma associação significativa com os desfechos na análise bivariada foram inseridas no modelo multivariado, mas apenas as que apresentaram significância estatística foram mantidas no mesmo. A técnica de regressão linear multivariada é utilizada para analisar o relacionamento entre uma única variável dependente e várias variáveis independentes. Desse modelo podemos retirar o coeficiente angular (b), que é um valor numérico que estima o efeito direto de uma variável independente sobre o desfecho em estudo. Além disso, é possível calcular o coeficiente de regressão padronizado (B), que permite uma comparação direta entre os efeitos das variáveis independentes, pois desconsidera a unidade da variável. Ainda é possível calcular o coeficiente de determinação $\left(R^{2}\right)$, que analisa o poder de explicação do conjunto de variáveis sobre o desfecho em estudo ${ }^{14}$.

O nível de significância adotado foi de $5 \%(\mathrm{p} \leq 0,05)$ e as análises foram realizadas no programa SPSS versão 21.0.

\section{RESULTADOS E DISCUSSÃo}

Participaram desta pesquisa 62 Agentes Comunitários de Saúde, com idade entre 23 e 61 anos, sendo 58 (93,5\%) mulheres e 4 (6,5\%) homens. Todos os participantes afirmaram ter participado do curso introdutório de ACS e ter reuniões de equipe sistemáticas.

Quanto à escolaridade, 1 deles (1,6\%) tinha ensino fundamental incompleto, 3 deles $(4,8 \%)$ têm o ensino fundamental completo, 14 deles $(22,6 \%)$ indicam ensino médio incompleto, $34(54,8 \%)$ citam ensino médio completo, 9 (14,5\%) têm ensino superior incompleto e 1 deles $(1,6 \%)$ indica ensino superior completo.

A maioria dos trabalhadores, mais especificamente 50 deles $(80,6 \%)$, era casada ou morava junto com um parceiro, sendo que $51(82,3 \%)$ residiam em domicílios próprios, 5 (8,1\%) em domicílios alugados e $6(9,7 \%)$ em domicílios cedidos. A média de tempo residindo na área em que trabalha variou de 9,3 a 28 anos. A renda familiar média foi de $\mathrm{R} \$ 2.949,34$. Os dados demonstram similaridade com outros estudos que objetivaram traçar perfis desta população, com exceção da caracterização da renda familiar, que se mostrou relativamente mais alta ${ }^{15,16,17}$.

O tempo médio do exercício do trabalho remunerado durante a vida foi de 15,5 anos e o tempo médio do exercício da função de ACS foi de 5 anos. Uma expressiva maioria, 59 participantes $(96,7 \%)$, cita já ter 
realizado trabalhos administrativos em sua unidade, enquanto que outros $61(98,4 \%)$ afirmam ter lidado com metas de produtividade. Segundo os ACSs, a maioria das equipes de ESF, mais especificamente 48 delas $(77,4 \%)$, está completa. Referentemente à disponibilidade de material de trabalho, 32 ACSs $(52,5 \%)$ referem não possuir materiais adequados, sendo que 13 deles (21\%) afirmam ter faltado ao trabalho por motivo de doença e 33 deles $(54,1 \%)$ fazem uso de medicamentos contínuos.

O instrumento utilizado (WHOQOL-BREF) mostrou características satisfatórias de consistência interna, validade discriminante, validade de critério, validade concorrente e fidedignidade teste-reteste. Alia um bom desempenho psicométrico com praticidade de uso, o que lhe coloca como uma alternativa útil para ser usada em estudos que se propõem a avaliar qualidade de vida ${ }^{18}$.

Através da Tabela 1, observa-se que o domínio mais afetado nos agentes comunitários é o ambiente, com média significativamente menor quando comparado com os demais $(\mathrm{p}=0,001)^{19,20,21}$.

Tabela 1 - Escores de qualidade de vida conforme WHOQOL-BREF

\begin{tabular}{lcc}
\multicolumn{1}{c}{ Escores } & Média \pm DP & Mínimo - Máximo \\
\hline Físico & $68,9 \pm 16,3$ & $21,4-92,9$ \\
\hline Psicológico & $67,6 \pm 18,0$ & $12,5-95,8$ \\
\hline Social & $70,2 \pm 18,0$ & $25,0-100$ \\
\hline Ambiente & $62,1 \pm 11,2$ & $34,4-87,5$ \\
\hline Geral & $65,3 \pm 19,0$ & $0,0-100$ \\
\hline
\end{tabular}

Fonte: elaborada pelo autor.

Houve associação positiva estatisticamente significativa entre o domínio psicológico com o nível de escolaridade $\left(\mathrm{r}_{\mathrm{s}}=0,255 ; \mathrm{p}=0,046\right)$ e renda familiar mensal ( $r s=0,313 ; \mathrm{p}=0,014$ ), ou seja, quanto maior o nível de escolaridade e renda familiar, maiores os escores de qualidade de vida nesse domínio (Tabela 2). Também houve associação positiva estatisticamente significativa entre o domínio ambiente com renda familiar mensal ( $r s=0,346 ; \mathrm{p}=0,006)$, sendo que quanto maior a renda familiar, maiores os escores de qualidade de vida nesse domínio. Tais achados estão de acordo com análises estabelecidas pelo Índice de Desenvolvimento Humano (IDH) enquanto indicador sintético de qualidade de vida que, de forma simplificada, soma e divide por três os níveis de renda, saúde e educação de determinada população. A renda é avaliada pelo Produto Interno Bruto (PIB) real per capita, a saúde é avaliada pela esperança de vida ao nascer, e a educação, pela taxa de alfabetização de adultos e taxas de matrículas nos níveis primário, secundário e terciário combinados. Renda, educação e saúde seriam atributos com igual importância como expressão das capacidades humanas ${ }^{22}$.

Por fim, houve associação negativa estatisticamente significativa entre o escore geral com o número 
de famílias cadastradas na microárea ( $\mathrm{r}=-0,275$; $\mathrm{p}=0,030$ ), ou seja, quanto maior o número de famílias cadastradas na microárea, menor o escore de qualidade de vida geral. O grande número de famílias cadastradas por agente dificulta a realização das visitas mensais a, aproximadamente, 750 pessoas (podendo variar de acordo com a realidade local) ${ }^{23}$, fazendo com que o trabalhador priorize famílias que necessitam de um maior acompanhamento, como aquelas com pessoas em tratamento médico, com tuberculose, AIDS, doença mental, gestantes, puérperas e crianças em risco nutricional ${ }^{24}$.

Tabela 2 - Associação dos escores de qualidade de vida com os dados contínuos e ordinais através dos coeficientes de correlação de Pearson ou Spearman

\begin{tabular}{|c|c|c|c|c|c|}
\hline Variáveis & Físico & Psicológico & Social & Ambiente & Geral \\
\hline Idade (anos) ${ }^{\text {(a) }}$ & $-0,053$ & 0,113 & $-0,069$ & 0,087 & 0,025 \\
\hline № de filhos ${ }^{(b)}$ & $-0,087$ & $-0,078$ & $-0,171$ & $-0,195$ & $-0,043$ \\
\hline Nível de escolaridade ${ }^{(b)}$ & 0,217 & $0,255^{\star}$ & $-0,037$ & 0,128 & 0,124 \\
\hline № de cômodos na residência ${ }^{(b)}$ & $-0,161$ & $-0,055$ & $-0,026$ & $-0,057$ & $-0,053$ \\
\hline $\begin{array}{l}\text { Tempo que mora na área em que } \\
\text { trabalha }{ }^{(b)}\end{array}$ & $-0,023$ & $-0,138$ & 0,107 & 0,026 & 0,000 \\
\hline № de pessoas que moram na casa ${ }^{(b)}$ & 0,052 & 0,012 & 0,114 & $-0,151$ & $-0,036$ \\
\hline Renda familiar mensal (b) & 0,115 & $0,313^{*}$ & 0,197 & $0,346^{* *}$ & 0,201 \\
\hline $\begin{array}{l}\text { Tempo que exerce trabalho remuner- } \\
\text { ado }{ }^{(b)}\end{array}$ & 0,101 & 0,179 & $-0,143$ & 0,128 & 0,118 \\
\hline $\begin{array}{l}\text { Tempo que exerce a função de agente } \\
\text { comunitário }^{(b)}\end{array}$ & $-0,037$ & $-0,004$ & 0,011 & $-0,060$ & $-0,107$ \\
\hline $\begin{array}{l}\text { № de famílias cadastradas na mi- } \\
\text { croárea }\end{array}$ & $-0,087$ & $-0,170$ & $-0,163$ & $-0,117$ & $-0,275^{*}$ \\
\hline $\begin{array}{l}\text { Tempo após o início que fez o curso } \\
\text { introdutório }\end{array}$ & $-0,034$ & $-0,048$ & 0,113 & $-0,017$ & $-0,051$ \\
\hline
\end{tabular}

(a) coeficiente de correlação de Pearson; ${ }^{(b)}$ coeficiente de correlação de Spearman; * $\mathrm{p}<0,05 ;{ }^{* *} \mathrm{p}<0,01$

A Tabela 3 apresenta a associação dos escores de qualidade de vida com os dados nominais. Os escores médios no domínio físico foram significativamente maiores nos profissionais que têm à disposição o material necessário para o seu trabalho $(\mathrm{p}=0,005)$, não faltam ao trabalho por motivo de doença $(\mathrm{p}=0,018) \mathrm{e}$ não fazem uso de medicamentos $(\mathrm{p}<0,001)$. Outros estudos apontam para o impacto que a falta de materiais traz para a vida dos trabalhadores, tanto para cargas físicas, no sentido de estarem se expondo a fatores de risco, quanto para cargas emocionais, quando falamos da falta de reconhecimento e 
valorização $0^{25,26,27}$.

Em relação aos escores médios no domínio psicológico e geral, esses foram significativamente maiores nos profissionais que não faltam ao trabalho por motivo de doença $(\mathrm{p}=0,012$ e $\mathrm{p}=0,002$, respectivamente) e não fazem uso de medicamentos ( $\mathrm{p}<0,001$ e $\mathrm{p}=0,001$, respectivamente). Esse dado está de acordo com pesquisa realizada com o intuito de analisar o absenteísmo relacionado à doença junto aos Agentes Comunitários de Saúde de Curitiba e de classificar as principais doenças relacionadas às licenças médicas, que mostrou que o número de absenteísmo-doença entre os ACSs encontrase elevado. Os escores médios no domínio social foram significativamente mais elevados nos agentes comunitários que não faltam ao trabalho por motivo de doença $(\mathrm{p}<0,001)^{28}$.

Enfim, os escores médios no domínio ambiente foram significativamente mais elevados nos agentes comunitários que não fazem uso de medicamentos $(\mathrm{p}=0,022)$. Esse dado corrobora com a pesquisa realizada com profissionais de uma equipe de ACSs de uma USF do município de Piracicaba, que tinha como objetivo compreender a relação entre as queixas de sofrimento e as condições de trabalho dos ACSs a fim de propor medidas para modificá-las ${ }^{29}$.

Conforme a Tabela 4 , as variáveis que permaneceram significativamente associadas com o domínio físico após o ajuste foram: ter à disposição o material necessário para o trabalho, fazer uso de medicamentos e ser da religião evangélica. Agentes comunitários que têm à disposição o material necessário para o trabalho apresentam, em média, 7,07 pontos a mais nesse domínio. No entanto, profissionais que fazem uso de medicamentos apresentam uma redução, em média, de 11,6 pontos no escore do domínio físico e os que citam ser da religião evangélica apresentam uma redução, em média, de 8,43 pontos nesse escore quando comparados aos de religião católica.

Conforme coeficiente de determinação $\left(\mathrm{R}^{2}\right)$, essas três variáveis em conjunto explicam 43,7\% da variabilidade das respostas nesse domínio. Através do coeficiente padronizado (B), a variável que mais fortemente está associada com esse domínio é fazer uso de medicamentos. Os dados relativos aos materiais e ao uso de medicamentos são reafirmados em diversas pesquisas ${ }^{25,26,27,28,29}$.

A implicação religiosa, enquanto fator determinante relacionado ao domínio físico, não foi encontrada na literatura atual, porém várias pesquisas denotam o papel da religiosidade do ACS, destacando a importância das crenças pessoais, sua influência no vínculo com os usuários e o desempenho do seu papel. Além disso, os valores culturais e religiosos podem influenciar na concepção de saúde do indivíduo ${ }^{19,24,30}$.

As variáveis que permaneceram significativamente associadas ao domínio psicológico após o ajuste foram: renda familiar mensal, faltar ao trabalho por motivo de doença e fazer uso de medicamentos. Agentes comunitários que têm uma renda de $\mathrm{R} \$$ 500,00 ou mais apresentam, em média, 2,22 pontos a mais nesse domínio. No entanto, profissionais que faltam ao trabalho por motivo de doença têm, em média, uma redução de 10 pontos nesse domínio. Por fim, os que fazem uso de medicamentos apresentam uma redução, em média, de 12,3 pontos no escore do domínio psicológico $22,28,31$.

Conforme coeficiente de determinação $\left(\mathrm{R}^{2}\right)$, essas três variáveis em conjunto explicam $38,7 \%$ da variabilidade das respostas no domínio físico. Novamente, através do coeficiente padronizado (B), a variável que mais fortemente está associada a esse domínio é fazer uso de medicamentos ${ }^{31}$.

Em relação ao domínio social, somente faltar ao trabalho por motivo de doença permaneceu associado estatisticamente a esse desfecho. Agentes comunitários que faltam ao trabalho por motivo de doença têm, em média, uma redução de 19,8 pontos nesse domínio. Conforme coeficiente de determinação $\left(\mathrm{R}^{2}\right)$, essa variável explica $20,4 \%$ da variabilidade das respostas no domínio social. Tal achado pode ser associado a variáveis estudadas 
por pesquisas que apontam o fato de a maioria dos trabalhadores serem mulheres e terem complicações na gravidez e no puerpério, além de estarem sobrecarregadas por cuidarem dos filhos e realizarem os afazeres domésticos ${ }^{32,33,34}$. Outra pesquisa aponta para a associação do achado ao fato de que, conforme o avanço da idade, há também uma maior probabilidade de os trabalhadores apresentarem morbidades ${ }^{35}$.

Tabela 3 - Associação dos escores de qualidade de vida com os dados nominais através do teste t-Student ou Análise de Variância (ANOVA)

\begin{tabular}{|c|c|c|c|c|c|}
\hline Variáveis & $\begin{array}{c}\text { Físico } \\
\text { Média } \pm \text { DP }\end{array}$ & $\begin{array}{l}\text { Psicológico } \\
\text { Média } \pm \text { DP }\end{array}$ & $\begin{array}{c}\text { Social } \\
\text { Média } \pm \text { DP }\end{array}$ & $\begin{array}{l}\text { Ambiente } \\
\text { Média } \pm \text { DP }\end{array}$ & $\begin{array}{c}\text { Geral } \\
\text { Média } \pm \text { DP }\end{array}$ \\
\hline \multicolumn{6}{|l|}{ Sexo $(c)$} \\
\hline Masculino & $71,4 \pm 3,6$ & $68,1 \pm 19,7$ & $77,8 \pm 24,1$ & $69,8 \pm 1,8$ & $62,5 \pm 21,7$ \\
\hline Feminino & $68,7 \pm 16,7$ & $67,6 \pm 18,1$ & $69,8 \pm 17,8$ & $61,8 \pm 11,4$ & $65,5 \pm 19,0$ \\
\hline \multicolumn{6}{|l|}{ Cor $(c)$} \\
\hline Branca & $69,8 \pm 15,6$ & $68,5 \pm 16,0$ & $70,5 \pm 17,5$ & $62,9 \pm 10,7$ & $66,2 \pm 18,6$ \\
\hline Não branca & $57,9 \pm 21,9$ & $57,7 \pm 35,0$ & $66,7 \pm 25,7$ & $54,1 \pm 14,6$ & $55,0 \pm 22,7$ \\
\hline \multicolumn{6}{|c|}{ Casado/Mora junto (c) } \\
\hline Sim & $69,7 \pm 15,2$ & $68,8 \pm 16,4$ & $71,3 \pm 18,2$ & $63,4 \pm 10,3$ & $66,3 \pm 18,3$ \\
\hline Não & $65,2 \pm 20,9$ & $62,4 \pm 23,6$ & $65,3 \pm 17,0$ & $57,1 \pm 13,9$ & $61,5 \pm 22,3$ \\
\hline \multicolumn{6}{|l|}{ Religião (d) } \\
\hline Católica & $73,5 \pm 11,5 b$ & $70,5 \pm 15,5$ & $71,7 \pm 16,3$ & $64,1 \pm 9,5$ & $69,7 \pm 15,3$ \\
\hline Evangélica & $66,1 \pm 18,2 \mathrm{ab}$ & $64,0 \pm 20,1$ & $70,1 \pm 17,6$ & $59,1 \pm 12,4$ & $61,9 \pm 19,8$ \\
\hline Espírita & $51,2 \pm 16,5 \mathrm{a}$ & $70,8 \pm 4,2$ & $55,6 \pm 33,7$ & $65,6 \pm 3,1$ & $62,5 \pm 12,5$ \\
\hline Não tem & $66,1 \pm 17,7 \mathrm{ab}$ & $72,9 \pm 2,9$ & $75,0 \pm 0,0$ & $64,1 \pm 2,2$ & $75,0 \pm 17,7$ \\
\hline \multicolumn{6}{|l|}{ Moradia (d) } \\
\hline Própria & $69,6 \pm 16,8$ & $68,2 \pm 17,5$ & $69,8 \pm 17,9$ & $62,8 \pm 11,1$ & $64,7 \pm 19,2$ \\
\hline Alugada & $61,4 \pm 17,2$ & $59,2 \pm 30,2$ & $76,7 \pm 27,3$ & $56,3 \pm 16,4$ & $70,0 \pm 24,4$ \\
\hline Cedida & $69,0 \pm 11,0$ & $69,2 \pm 8,3$ & $68,1 \pm 11,1$ & $62,0 \pm 7,5$ & $66,7 \pm 15,1$ \\
\hline \multicolumn{6}{|c|}{ Você tem à sua disposição o material necessário para o seu trabalho? (c) } \\
\hline $\operatorname{Sim}$ & $74,4 \pm 9,8^{* *}$ & $70,7 \pm 14,0$ & $71,6 \pm 17,4$ & $63,9 \pm 9,9$ & $69,1 \pm 15,9$ \\
\hline Não & $62,4 \pm 19,9$ & $64,3 \pm 21,5$ & $69,0 \pm 19,0$ & $60,1 \pm 12,5$ & $61,2 \pm 21,7$ \\
\hline \multicolumn{6}{|c|}{ Sua equipe de ESF é completa? (c) } \\
\hline $\operatorname{Sim}$ & $67,7 \pm 17,0$ & $66,2 \pm 17,7$ & $69,3 \pm 18,6$ & $61,8 \pm 11,1$ & $63,5 \pm 19,8$ \\
\hline Não & $72,7 \pm 13,8$ & $72,3 \pm 18,8$ & $73,2 \pm 16,1$ & $63,4 \pm 11,9$ & $71,4 \pm 15,1$ \\
\hline \multicolumn{6}{|c|}{ Você faltou ao trabalho no último mês por motivo de doença? (c) } \\
\hline $\operatorname{Sim}$ & $56,3 \pm 20,5^{*}$ & $56,6 \pm 23,1^{*}$ & $54,5 \pm 19,1^{\star \star \star}$ & $59,1 \pm 11,7$ & $51,0 \pm 24,7^{\star *}$ \\
\hline Não & $72,2 \pm 13,4$ & $70,5 \pm 15,4$ & $74,3 \pm 15,4$ & $63,0 \pm 11,1$ & $69,1 \pm 15,3$ \\
\hline \multicolumn{6}{|c|}{ Você está fazendo uso de medicamentos? (c) } \\
\hline $\operatorname{Sim}$ & $61,3 \pm 15,7^{\star \star \star}$ & $60,1 \pm 17,7^{\star \star *}$ & $66,2 \pm 19,8$ & $59,2 \pm 10,2^{*}$ & $57,6 \pm 18,2^{\star *}$ \\
\hline Não & $77,7 \pm 12,6$ & $75,7 \pm 14,3$ & $74,1 \pm 14,8$ & $65,7 \pm 11,7$ & $73,7 \pm 16,1$ \\
\hline
\end{tabular}


As variáveis que permaneceram significativamente associadas com o domínio ambiente após o ajuste foram: renda familiar mensal e fazer uso de medicamentos. Agentes comunitários que têm uma renda de $R \$ 500,00$ ou mais apresentam, em média, 1,57 pontos a mais nesse domínio ${ }^{22}$. No entanto, profissionais que fazem uso de medicamentos apresentam uma redução, em média, de 7,05 pontos no escore do domínio ambiente. Conforme coeficiente de determinação $\left(\mathrm{R}^{2}\right)$, essas duas variáveis em conjunto explicam $19,6 \%$ da variabilidade das respostas no domínio ambiente. Novamente, através do coeficiente padronizado (B), a variável que mais fortemente está associada a esse domínio é fazer uso de medicamentos ${ }^{30}$.
Por fim, as variáveis que permaneceram significativamente associadas ao escore geral do WHOQOL-BREF após o ajuste foram: faltar ao trabalho por motivo de doença e fazer uso de medicamentos. Agentes comunitários que faltam ao trabalho por motivo de doença têm, em média, uma redução de 12,3 pontos nesse domínio, enquanto que os que fazem uso de medicamentos apresentam uma redução, em média, de 12,9 pontos no escore geral do instrumento ${ }^{28,31,32,33,34}$. Conforme coeficiente de determinação $\left(R^{2}\right)$, essas duas variáveis em conjunto explicam $31,2 \%$ da variabilidade das respostas no escore geral. Mais uma vez, através do coeficiente padronizado (B), a variável que mais fortemente está associada a esse domínio é fazer uso de medicamentos.

Tabela 4 - Análise de Regressão Linear Multivariada para avaliar fatores independentemente associados aos domínios do WHOQOL-BREFFonte: elaborada pelo autor.

\begin{tabular}{|c|c|c|c|c|}
\hline Variáveis & b (IC 95\%) & Beta $(B)$ & $\mathbf{p}$ & $\mathbf{R}^{2}$ \\
\hline Domínio Físico & & & & $43,7 \%$ \\
\hline Tem à sua disposição o material necessário para o seu trabalho & $7,07(0,30$ a 13,8$)$ & 0,229 & 0,041 & \\
\hline Fazendo uso de medicamentos & $-11,6(-18,6$ a $-4,6)$ & $-0,375$ & 0,002 & \\
\hline Religião evangélica & $-8,43(-15,4$ a $-1,4)$ & $-0,263$ & 0,019 & \\
\hline Domínio Psicológico & & & & $38,7 \%$ \\
\hline Renda familiar mensal (a cada $\mathrm{R} \$ 500,00$ ) & $2,22(0,33$ a 4,11$)$ & 0,250 & 0,022 & \\
\hline Faltou ao trabalho no último mês por motivo de doença & $-10,0(-19,9$ a $-0,17)$ & $-0,232$ & 0,046 & \\
\hline Fazendo uso de medicamentos & $-12,3(-20,4$ a $-4,19)$ & $-0,354$ & 0,004 & \\
\hline Domínio Social & & & & $20,4 \%$ \\
\hline Faltou ao trabalho no último mês por motivo de doença & $-19,8(-29,9$ a 9,72$)$ & $-0,452$ & $<0,001$ & \\
\hline Domínio Ambiente & & & & $19,6 \%$ \\
\hline Renda familiar mensal (a cada $\mathrm{R} \$ 500,00$ ) & $1,57(0,23$ a 2,91$)$ & 0,279 & 0,023 & \\
\hline Fazendo uso de medicamentos & $-7,05(-12,3$ a $-1,82)$ & $-0,322$ & 0,009 & \\
\hline Geral & & & & $31,2 \%$ \\
\hline Faltou ao trabalho no último mês por motivo de doença & $-12,3(-24,3$ a $-0,23)$ & $-0,260$ & 0,046 & \\
\hline Fazendo uso de medicamentos & $-12,9(-22,1$ a $-3,82)$ & $-0,344$ & 0,006 & \\
\hline
\end{tabular}

b=coeficiente angular (representa o efeito de cada fator sobre cada desfecho); IC95\%=intervalo de 95\% de confiança; B=coeficiente de regressão padronizado; $\mathrm{R}^{2}=$ coeficiente de determinação 


\section{CONCLUSÕES}

A execução desta pesquisa pôde confirmar estatisticamente o impacto do processo de trabalho nos diferentes domínios da qualidade de vida.

Foi possível verificar que o domínio mais afetado pelo trabalho é o ambiente, estando diretamente ligado às variáveis de renda e de uso de medicamentos. As variáveis que permaneceram significativamente associadas a esse domínio após o ajuste foram: renda familiar mensal e fazer uso de medicamentos. Os escores médios no domínio ambiente foram significativamente mais elevados nos agentes comunitários que não fazem uso de medicamentos.

O domínio psicológico demonstrou-se afetado pela renda familiar, pelo nível de escolaridade, pelo fato de faltar ao trabalho por motivo de doença e de fazer uso de medicamentos. Os escores médios no domínio psicológico e geral foram significativamente maiores nos profissionais que não faltam ao trabalho por motivo de doença e não fazem uso de medicamentos.

O domínio físico está relacionado a ter à disposição o material necessário para o trabalho, fazer uso de medicamentos e ser da religião evangélica. Os escores médios foram significativamente maiores nos profissionais que têm à disposição o material necessário para o seu trabalho, não faltam ao trabalho por motivo de doença e não fazem uso de medicamentos

Em relação ao domínio social, somente faltar ao trabalho por motivo de doença permaneceu associado estatisticamente a esse desfecho.

O escore geral de qualidade de vida recebeu influência do número de famílias cadastradas. Por fim, as variáveis que permaneceram significativamente associadas ao escore geral do WHOQOL-BREF após o ajuste foram: faltar ao trabalho por motivo de doença e fazer uso de medicamentos.

O estudo gerou reflexões acerca do processo de trabalho dos Agentes Comunitários de Saúde do município de Campo Bom, no Rio Grande do Sul.
Espera-se que com o levantamento e a publicação desses dados seja possível sensibilizar o poder público e fomentar novas pesquisas que busquem maior reconhecimento e valorização destes profissionais.

\section{REFER̂̂ENIAS}

1. Dejours C, Abdoucheli E, Jayet C. Psicodinâmica do trabalho: contribuição da escola dejouriana à análise da relação prazer, sofrimento e trabalho. São Paulo: Atlas; 1994.

2. Brasil. Lei $\mathrm{n}^{-}$11.350, de 5 de outubro de 2006 . Regulamenta o § 5 do art. 198 da Constituição, dispõe sobre o aproveitamento de pessoal amparado pelo parágrafo único do art. 2ำ da Emenda Constitucional nº 51, de 14 de fevereiro de 2006, e dá outras providências. Diário Oficial da União, 2006.

3. Nunes MO, Trad LB, Almeida BA, Homem CR, Melo MCIC. O agente comunitário de saúde: construção da identidade desse personagem híbrido e polifônico. Cad Saúde Pública. 2002;18:1639-46.

4. Marx, K. O capital: crítica da economia política - Livro 1: o processo de produção do capital. São Paulo: Difel; 1982.

5. Vasconcellos NPC, Costa-Val R. Avaliação da qualidade de vida dos agentes comunitários de saúde de Lagoa Santa - MG. Revista de APS. 2008;11(1):17-28.

6. Mendes R, Dias EC. Da medicina do trabalho à saúde do trabalhador. Rev. Saúde Pública. 1991;25(5):341-349.

7. Kirogi MS. Qualidade de vida no trabalho e suas diversas abordagens. Rev Ciencias Gerenciais. 2008;12(16): 49-62.

8. Amatuzzi MM. O significado da psicologia humanista, posicionamentos filosóficos implícitos. Arquivos Brasileiros de Psicologia. 1989;41(4): 88-95.

9. Kluthcovsky ACGC et al. Qualidade de vida de agentes comunitários de saúde. Rev Psiquiatr RS. 2007;29(2):176183.

10. Davis K, Newstrom JW. Comportamento humano no trabalho: uma abordagem psicológica. São Paulo: Pioneira; 1992. 
11. Rodrigues MVC. Qualidade de vida no trabalho: evolução e análise no nível gerencial. Rio de Janeiro: Vozes; 1994.

12. Tannenbaum AS. Psicologia social das organizações do trabalho. São Paulo: Atlas; 1976.

13. Aquino CP. Administração de recursos humanos: uma introdução. São Paulo: Atlas; 1979.

14. Hair JF, Anderson RE, Black WC. Multivariate Data Analysis. New Jersey, USA: Prentice Hall; 1998.

15. Lino MM, Lanzoni GMM, Albuquerque GL, Schveitzer MC. Perfil socioeconômico, demográfico e de trabalho dos agentes comunitários de saúde. Cogitare Enferm. 2012;17(1):57-64.

16. Santos KT, Moimaz SAS, Arcieri RM, Carvalho ML. Agentes comunitários de saúde: perfil adequado à realidade do programa de saúde da família? Ciên. Saúde Colet [Internet]. 2011 [acesso em 2015 Jul 15]. Disponível em: www.scielo.br/pdf/csc/v16s1/a35v16s1.pdf

17. Silva ATC, Menezes PR. Esgotamento profissional e transtornos mentais comuns em agentes comunitários de saúde. Rev. Saúde Pública. 2008;42(5):921-9.

18. Fleck MPA et al. Aplicação da versão em português do instrumento abreviado de avaliação da qualidade de vida “WHOQOL-bref”. Rev. Saúde Pública. 2000;34(2):178-183.

19. Vasconcellos NPC, Costa-Val R. Avaliação da qualidade de vida dos agentes comunitários de saúde de Lagoa SantaMG. Rev. de APS. 2008;11(1):17-28.

20. Ursine BL, Trelha CS, Nunes EFPA. O Agente Comunitário de Saúde na Estratégia de Saúde da Família: uma investigação das condições de trabalho e da qualidade de vida. Rev. Bras. Saúde Ocup. 2010;35(122 ):327-339.

21. Mascarenhas CHM, Prado FO, Fernandes MH. Fatores associados à qualidade de vida de agentes comunitários de saúde. Ciên. Saúde Colet. 2013;18(5):1375-86.

22. Minayo MCS, Hartz ZMA, Buss PM. Qualidade de vida e saúde: um debate necessário. Ciên. Saúde. Colet. 2000;5(1):7-18

23. Brasil. Ministério da Saúde. Secretaria Executiva. Programa Agentes Comunitários de Saúde, PACS. Brasília-DF: Ministério da Saúde; 2001.
24. Ferraz L, Aerts DRGC. O cotidiano de trabalho do agente comunitário de saúde no PSF em Porto Alegre. Ciên. Saúde Colet. 2005;10(2):347-355.

25. Trindade LDL, Gonzales RMB, Beck CLC, Lautert L. Cargas de trabalho entre os agentes comunitários de saúde. Rev. Gaúcha Enferm. 2007;28(4):473-479.

26. Oliveira AR et al. Satisfação e limitação no cotidiano de trabalho do agente comunitário de saúde1. Rev. Eletrônica Enferm. 2010; 12(1):28-36.

27. Bezerra AFB, Espírito Santo ACG, Batista Filho M. Concepções e práticas do agente comunitário na atenção à saúde do idoso. Rev. Saúde Pública. 2005;39(5):809-815.

28. Kami MT et al. Absenteísmo relacionado a doenças em agente comunitário de saúde: novo olhar do enfermeiro. $61^{\circ}$ Congresso Brasileiro de Enfermagem; 2009; Fortaleza, CE [Internet]. [acesso em 05 Jun 2016]. Disponível em: http:// www.abeneventos.com.br/anais_61cben/files/02608.pdf

29. Vilela RAG, Silva RC, Jackson Filho JM. Poder de agir e sofrimento: estudo de caso sobre Agentes Comunitários de Saúde. Rev. Brasileira de Saúde Ocupacional. 2010; 122(35): 289-302.

30. Scliar M. História do conceito de saúde. Physis. 2007;17(1):29-41.

31. Tomasi E et al. Condições de trabalho e automedicação em profissionais da rede básica de saúde da zona urbana de Pelotas, RS. Rev.. Bras. Epidemiol. 2007;10(1):66-74.

32. Silva DMPP, Marziale MHP. Absenteísmo de trabalhadores de enfermagem em um hospital universitário. Rev. Lat Am Enfermagem. 2000;8(5):44-51.

33. Reis RJ, La Rocca PF et al. Fatores relacionados ao absenteísmo por doença em profissionais de enfermagem. Rev. Saúde Pública. 2003;37(5):616-723.

34. Montes I, Claros MG, Belo Horizonte MG. Absenteísmo relacionado a doenças entre membros da equipe de enfermagem de um hospital escola. Rev. Bras. Enferm. 2009;62(1):38-44.

35. Nascimento GM. Estudo do absenteísmo dos trabalhadores de enfermagem em uma Unidade Básica e Distrital de saúde do município de Ribeirão Preto [dissertação]. [São Paulo (SP)]: Escola de Enfermagem da USP, Universidade de São Paulo; 2003. 143 p. 